\title{
Editorial
}

\section{Long-drawn-out story}

The referee suggested that we “... better define and explain 'alternate segregation' and 'adjacent-1 segregation'. A simple figure illustrating these alternatives would help a great deal”. Back in 1964, La Chance, Riemann, and Hopkins (Genetics 49 959-972) wearied that '... chromosome pairing ... in a translocation heterozygote ... (has) been diagrammed and discussed in many publications ...' Geneticists working with translocations must continue to bear the cross of making 'alt / adj-1' figures afresh.

La Chance et al. reported an autosomal dominant mutant strain of the screw-worm fly Cochliomyia hominivorax. Females lay eggs in the wounds of warm-blooded animals, the maggots hatch and burrow through a feast of warm flesh, producing foul-smelling pus that attracts more flies. Mature larvae drop from the wound, pupate in the soil, bluish-green adults eclose, mate, and repeat nature's 'red in ovipositor and mouth-hook' cycle. In mutant flies, a wing segment called the R-cell was blackened by pigmentation. The Brc (black R-cell) mutant phenotype was associated with a reciprocal translocation. Several generations of $\mathrm{Brc} \times \mathrm{Brc}$ crosses failed to establish a pure-breeding strain, suggesting that translocation-homozygotes were inviable. Remarkably, in each generation the ratio of Brc to wild-type flies was 3:1 instead of the expected 2:1 (1 lethal Brc/ $\mathrm{Brc}: 2 \mathrm{Brc} /+$ heterozygotes : $1+/+$ wild-type homozygote). They proposed that in Brc males alternate segregation is twice as frequent as adjacent-1, a kind of chromosomal meiotic drive.

Segregation Distorter (SD) of Drosophila melanogaster is the best-studied meiotic drive (Genetics 192 33-53, 2012). All sperm from $S D / S D^{+}$males pass on the $S D$-bearing chromosome-2 to their progeny. The $S d$ gene, in chromosome-2L, is a $3^{\prime}$ truncated version of the Ran GTPase activating protein (RanGAP) gene created by a tandem duplication of a segment of the RanGAP gene. The C-terminally truncated Sd-RanGAP protein is enzymatically active but lacks the wild-type RanGAP's localization signals. While wild-type RanGAP is tethered to the cytoplasmic side of the nuclear envelope, Sd-RanGAP accumulates in the nucleus and the cytoplasm. Mislocalization of Sd-RanGAP depletes nuclear RanGTP and impairs nuclear transport. $S D$ chromosomes are $S d R s p^{i}$ in genotype, whereas most $S D^{+}$chromosomes are $S d^{+} R s p^{s}$. The $R s p^{s}$ (Respondersensitive) locus in drive-sensitive $S d^{+}$spermatids has several hundred copies of a 240 bp satellite DNA repeat, whereas the $R s p^{i}$ allele in insensitive spermatids has very few or no satellite repeats. The following model was proposed for $R s p$ 's role (Genetics 193 771-784, 2013). Under non-distorting (i.e. SD $/ \mathrm{SD}^{+}$) conditions, precursor $R s p$ piRNA transcripts are exported from the nucleus, processed into mature piRNAs, and reenter the nucleus as part of a Rsp-piRNA-primed RNAprotein silencing complex (Rsp RNP) that silences the $R s p^{s}$ locus and enables $R s p^{s}$-bearing chromosomes to condense chromatin. Under distorting (i.e. SD/SD ${ }^{+}$) conditions, perturbation of nuclear transport retains the Rsp precursor piRNAs in the nucleus, and/or blocks nuclear entry of the Rsp RNP. Consequently, $R s p^{s}$ is not silenced, the $R s p^{s}$-bearing spermatids fail to condense chromatin, and are eliminated. In contrast, $R s p^{i}$ spermatids can condense chromatin in either distorting or nondistorting conditions.

In fungi, meiotic drive was shown to propel species divergence (eLife 3 e02630, 2014), and Spore-killer genes that kill meiotic products not containing them are known (PNAS 109 12093-12098, 2012; PLOS Genet. 10 e1004387, 2014). We were excited to report yet another drive: crosses heterozygous for Neurospora crassa translocations introgressed into N. tetrasperma make fewer homokaryotic ascospores following alternate than adjacent-1 segregation (G3 5 1263-1272, 2015). Admittedly a more recondite finding, our referee was only trying to help us to disseminate it.

Durgadas P Kasbekar Editor 